\title{
Prevention and treatment of cerebral palsy caused by intrapartum damage with novel hypoxia index
}

\begin{abstract}
Cerebral palsy is a big problem in fetal monitoring. Novel hypoxia index=the sum of all fetal heart rate deceleration durations ( $\mathrm{min}$ ) in fetal monitoring, which is divided by the lowest fetal heart rate (bpm), and multiplied by 100 . The hypoxia index of all 6 cerebral palsy cases was 25 or more, while the index of all 16 normal non cerebral palsy cases was 24 or less. Thus, cerebral palsy is prevented, if hyoxia index is 24 or less in the birth, and the newborn babies whose hypoxia index was 25 or more can be treated by early cerebral palsy therapy in neonatal stage. As the hypoxia index is adopted to all of deceleration patterns and continuous bradycardia because its calculation equation did not include lag-time, traditional but subjective early, late and variable deceleration pattern classification will change to numeric hypoxia index in fetal monitoring.
\end{abstract}

Volume 4 Issue 4 - 2018

\author{
Kazuo Maeda \\ Obstetrics \& Gynecology, Tottori University Medical School, \\ Japan
}

Correspondence: Kazuo Maeda, Honorary Professor, Obstetrics \& Gynecology, Tottori University Medical School, Yonago, Japan, Tel 8I-859-22-6856;

Email maedak@mocha.ocn.ne.jp

Keywords: Obstetrics, hypoxia index, cerebral palsy, diagnosis, hemorrhage, deceleration

\section{Introduction}

Cerebral palsy has been a big problem of Obstetrics \& Pediatrics, because there have been no reduction of cerebral palsy in the world, in spite of the progress and big efforts of intrapartum fetal monitoring in Obstetrics.

The author was afraid of the situation on the absence of numeric indicator to develop cerebral palsy in the guidelines of fetal monitoring, where diagnosis was based on subjective pattern classification of fetal heat rate (FHR) decelerations into early, late and variable, but objective numeric diagnosis was uncommon, however, the author noticed the paradoxical nature of late deceleration, which was diagnosed by visual recognition of later appearance of deceralation to uterine contraction wave.

\section{Methods}

The analysis of vague results of late FHR deceleration in the record of FHR and uterine contraction, that was cardio-toco-gram (CTG), where 3 typical late decelerations (LD) were recorded, where the LD was late to uterine contraction for 45 seconds and they were typical wave pattern of a LD according to Hon's Atlas. ${ }^{1}$ As 3 typical LDs were connected in an actocardiogram (ACG) of the patient in the birth process, the author expected a depressed neonate after a caesarean delivery according to textbooks of CTG, which listed neonatal asphyxia characterized low Apgar score lower than 7, which was recommended the early delivery. However, the neonate was fully vigorous. Apgar score was 9 and normal, which was close to 10 full points. Resuscitation was prepared but it was non-necessary (Figure 1).

However, another case of delivery gave the birth of severely depressed neonate after 50 minutes repetition of typical LDs due to maternal refusal of caesarean delivery, where the FHR baseline variability was lost, which was similar to anencephalic fetus (Figure 2). Apgar score was 3, severe brain damage and hemorrhage followed by death.

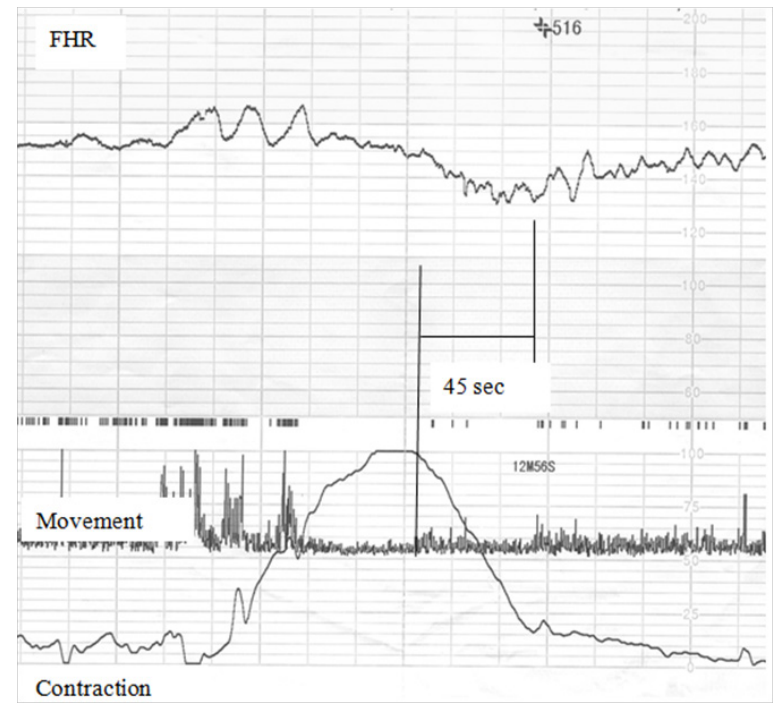

Figure I Typical late decelerations repeated for 3 times, while the neonate was normal and Apgar score was 9 and normal.
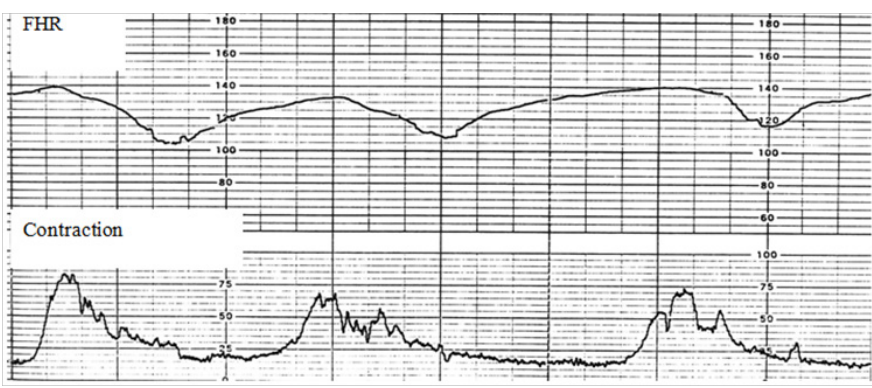

Figure 2 Another typical late decelerations repeated for 50 minutes. Baseline variability was lost, Apgar score was as low as 3, and brain damage was as heavy as brain hemorrhage.

In addition experts defined LD after its 15 minutes' repetition. Thus, 
the author understood that $\mathrm{LD}$ is ominous due to its frequent repetition but not due to the typical late appearing pattern. The vicious nature of LD is based on the repeating decelerations. It was important to clarify developing process of deceleration. The low $\mathrm{PaO}_{2}$, that is the hypoxia, stimulate parasympathetic center located in the medulla oblongata developing parasympathetic excitation (vagotonia), which decrease heart rate and develop bradycardia, of which transient appearance is the deceleration, namely, the deceleration is the expression of fetal hypoxia, thus the deceleration duration means transient hypoxia, and the lowest FHR indicates the intensity of hypoxia. So that, the hypoxia index was composed as the sum of deceleration durations (min) divided by the lowest FHR (bpm), and multiplied by 100 to keep the index an integer (Figure 3), thus hypoxia index is the sum of transient hypoxia expressing total sum of hypoxic duration, which will damage the fetus when the decelerations frequently repeats, but exhibits no hypoxic effect, if the sum of deceleration duration is shortly.

The hypoxia index utilize bradycardia instead of $\mathrm{PaO}_{2}$, because heart rate is fully parallel to $\mathrm{PaO}_{2}$, if the $\mathrm{PaO}_{2}$ is lower than $50 \mathrm{mmHg}$, and human fetal $\mathrm{PaO}_{2}$ was less than $50 \mathrm{mmHg} .{ }^{3}$ Cases whose outcome was diagnosed by pediatric doctor, and their intrapartum FHR curves were fully preserved in obstetrics, were collected to study hypoxia index. Collected cases were 22, including 6 cerebral palsy cases and 16 normal cases without cerebral palsy. The hypoxia index (HI) of 50 minutes LD repetition was 26, and the hypoxia indices of 6 cerebral palsy cases were 25 or more. The HI of 3 LDs was 6, and all HIs of normal outcome cases without cerebral palsy was 24 or less (Table 1).

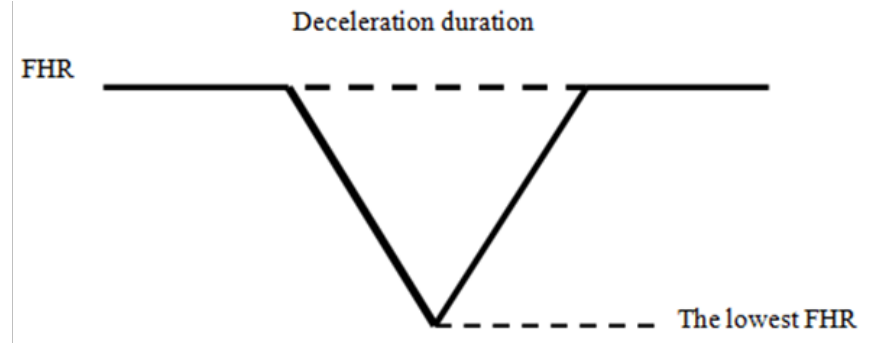

Figure 3 Determination of deceleration duration and the lowest FHR in the hypoxia index

Table I Fetal hypoxia index of all 6 cerebral palsy cases were 25 or more, and those of all 16 no cerebral palsy cases were 24 or less

\begin{tabular}{llll}
\hline \multirow{2}{*}{ Hypoxia index } & \multicolumn{3}{c}{ Cerebral palsy } \\
\cline { 2 - 4 } & $\mathbf{N}$ & Yes & No \\
\hline 25 or more & 6 & 6 & 0 \\
24 or less & 16 & 0 & 16 \\
\hline
\end{tabular}

\section{Discussion}

There was cerebral palsy in all 6 cases whose hypoxia index was 25 or more, while no cerebral palsy was found in all of 16 cases whose hypoxia index was 24 or less (Table1). Therefore, cerebral palsy will be prevented, if the hypoxia index is 24 or less during the labor process, according to the above retrospective study, namely, hypoxia index should be 24 or less during the birth process to prevent infantile cerebral palsy. ${ }^{3}$

As it was reported by Poseiro, ${ }^{4}$ late deceleration was caused by the loss of blood flow through placenta due to the compression of maternal iliac artery with hardly contracted heavy pregnant uterus in supine posture, and late deceleration disappeared after maternal lateral posture changed from supine one. Thus, the author recommends maternal lateral posture to left or right, when any deceleration is found on the FHR record in fetal monitoring, before the calculation of hypoxia index, namely, the deceleration will disappear before the calculation of hypoxia index, then continue lateral or sitting posture until the early delivery, or the vaginal delivery, if the threatening LD is removed by the posture change. The increasing hypoxia index should be carefully monitored by FHR monitoring, particularly by computerized FHR monitoring including FHR score, predicting neonatal Apgar score and $\mathrm{UApH},{ }^{5}$ hypoxia index and FHR frequency spectrum, detecting pathologic sinusoidal FHR, ${ }^{5}$ because the index is applied the other decelerations and continuous fetal bradycardia predicting hypoxicischemic encephalopathy (HIE), particularly, continuous bradycardia increases more rapidly than transient bradycardia. Be carefully watched the remained minutes until 24 of hypoxia index reported by coming computer to plan caesarean delivery.

If unfortunately, the hypoxia index increases pathologic level of hypoxia index, that is 25 or more predicting the cerebral palsy, the neonate can be treated by early treatment of the brain damage, including brain damage repairing with stem cells and growth factor, glucocorticoid, antiglutamate, free radical scavenger, erythropoietin, head hypothermia, etc, already known treatment by pediatrician, namely, hypoxia index also indicate the early treatment of cerebral palsy may promote the effect of broken neuronal fiber between cortex and spine.

\section{Conclusion}

Hypoxia index prevents cerebral palsy monitoring the progress of intrapartum hypoxia to perform early delivery. The late deceleration is treated changing maternal supine posture to lateral one. .Very early treatment of brain damage preceding cerebral palsy will be suggested by early diagnosis of brain damage with hypoxia index.

\section{Acknowledgements}

None.

\section{Conflicts of interest}

Author declares that there is none of the conflicts.

\section{References}

1. Hon EH. An Atlas of Fetal Heart Rate Patterns. USA: Harty Press; 1968.

2. Umezawa J. Studies on the relation between heart rate and $\mathrm{PaO}_{2}$ in hypoxic rabbit: a comparative study for fetal heart rate change in labor. Acta Obstet Gynecol. 1975;28:1203-1212.

3. Maeda K, Kimura S, Nakano H, et al. Pathophysiology of Fetus. Fukuoka; 1969.

4. Poseiro JJ, Mendez-Bauer C, Caldeyro-Barcia R, et al. Effect of uterine contractions on maternal blood flow through the placenta. Perinatal factors affecting human development, Paho Advisary Committee; 1969. p. $161-171$.

5. Maeda K, Noguchi Y, Matsumoto F, et al. Quantitative fetal heart rate evaluation without pattern classification: FHR score and artificial neural network analysis. In: Kurjak \& Chervenak editors. Textbook of Perinatal Medicine, Volume 2. UK: Informa; 2006. p. 1487-1495. 